\section{Gespräche und Verhandlungen zur UN-Klimarahmenkonvention}

\author{
Bonn, 15. - 26. Mai 2006 \\ von Gerhard Sardemann, ITAS
}

\section{Vorbemerkung}

Dieser Bericht über eine weitere Runde von Verhandlungen zur Klimarahmenkonvention in Bonn stammt nicht von einem Sitzungsteilnehmer, sondern fasst die Geschehnisse ,aus der Ferne" zusammen. Als Mitarbeiter der Informationsstelle Umweltforschung des Bundesministeriums für Bildung und Forschung, die bis zu ihrem Auslaufen im Jahr 1998 am ITAS angesiedelt war, gehörte es zu den Aufgaben des Autors, die Verhandlungen und gesellschaftlichen Debatten zur Klimarahmenkonvention, aber auch die Sitzungen des „Intergovernmental Panel on Climate Change“" (IPCC) zu beobachten und aus der Sicht der Forschungsförderung in Deutschland zu bewerten.

Das Geschehen während der Sitzungen kann inzwischen anhand von Aufzeichnungen im Internet in Wort, Bild und Ton nachvollzogen werden. Was dem Beobachter aus der Ferne allerdings fehlt, sind Eindrücke über die Gespräche und Stimmungen auf den Fluren, die zwischen den jeweiligen Verhandlungen und Veranstaltungen stattfanden. Aber auch dafür gibt es eigene Rubriken in den „Earth Negotiations Bulletins“ (hg. v. International Institute for Sustainable Development), die aktuell von wichtigen UN-Verhandlungen berichten. Informationen liefern auch die im Bereich Umwelt tätigen Nichtregierungsorganisationen (Environmental NGOs bzw. ENGOs) in ihrem Organ „ECO“, das vor allem in der Anfangsphase der Klimaverhandlungen vor über zehn Jahren für viele der teilnehmenden Parteien vor Ort ein wichtiges Hilfsmittel darstellte, sich überhaupt in der Vielzahl der während der Verhandlungen gemachten Vorschläge und deren Bedeutung zurechtzufinden. Während die ENGOs bislang die Mehrheit der an den Verhandlungen beteiligten Nichtregierungsorganisationen und vor allem der gelieferten Beiträge stellte, gab es bei den diesjährigen Verhandlungen in Bonn zum ersten Mal mehr Einwürfe von den „Business and Industry NGOs“" (BINGOs).

\section{Die unterschiedlichen Verhandlungs- stränge}

Bei diesem Treffen in Bonn handelte es sich um eine Veranstaltung zwischen zwei Vertragsstaatenkonferenzen zur Klimarahmenkonvention, die seit der ersten, 1995 in Berlin von Angela Merkel als Umweltministerin geleiteten Konferenz im regelmäßigen Turnus stattfinden. Mit den Verhandlungen begann die Umsetzung des Aktionsplanes, der auf der 11. Vertragsstaatenkonferenz zur Klimarahmenkonvention (COP11) und der 1. Konferenz der Vertragsparteien des Kioto-Protokolls (COP/MOP 1) Ende letzten Jahres in Montreal beschlossen worden war.

Die kanadische Umweltministerin Rona Ambrose ist seit Januar Präsidentin der Vertragsstaatenkonferenz. Schon zu Beginn der Gespräche in Bonn wurde von den UmweltNGOs gefordert, allerdings vergeblich, dass Ambrose ihr Mandat niederlegt. In Montreal habe die kanadische Regierung noch große Anstrengungen gezeigt, die Klimaverhandlungen voranzubringen. Die im Januar eingesetzte neue Regierung, der Ambrose angehört, mache dagegen keine Anstalten, den Anstieg der Treibhausgasemissionen in Kanada zu bremsen und, toleriere ein Verfehlen der im Kioto-Protokoll festgelegten Ziele sogar ausdrücklich. Wie könne man aus einer solchen Situation heraus Sitzungen leiten, in denen es u. a. um die „Bestrafung““ von Abweichungen von den Kioto-Zielen geht? Die kanadischen Verhandlungsführer wurden von ihrer Regierung zudem angewiesen, ambitioniertere Kioto-Ziele für die Zeit nach $2012 \mathrm{zu}$ verhindern Außerdem wird darüber spekuliert, dass Kanada der Asia-Pacific climate partnership (,AP6“ zusammen mit den USA, Japan und Australien sowie den Schwellenländern China, Indien und Südkorea), gewissermaßen einem Konkurrenzunternehmen zum Kioto-Protokoll, beitreten könnte. Damit machten vorweg ausgesprochene Warnungen vor eigenen Wegen und bilateralen Lösungen Sinn.

Insgesamt fanden in Bonn drei Sitzungen statt:

- die erste von vier Dialogsitzungen aller Vertragsstaaten der Klimarahmenkonvention zur Fortentwicklung des internationalen Klimaschutzregimes (15.-16. Mai 2006), 
- die erste Sitzung der Ad-hoc-Arbeitsgruppe zur Fortentwicklung des Kioto-Protokolls (AWG)(17.-25. Mai 2006) und

- die 24. Sitzung der Nebenorgane der Klimarahmenkonvention und des Kioto-Protokolls (18.-26. Mai 2006).

Daneben gab es Workshops zu den Themen der ökonomischen Diversifikation, zur Kohlenstoffabscheidung und -speicherung, Kohlenstoffspeicherung im Rahmen des „Clean Development Mechanism" und Emissionsminderung bei Land- und Forstwirtschaft und in Bezug auf ländliche Entwicklung.

\subsection{Die Dialogsitzung „Open Dialogue“}

„Open Dialogue“ ist ein Schlüsselbegriff des Montrealer Aktionsplans und steht für die Anstrengungen, auch die USA und Australien wieder ins Boot zu holen, die das Kioto-Protokoll nicht unterzeichnet haben. Der Dialog über die weitere, langfristige Ausgestaltung der Verpflichtungen unter der Klimarahmenkonvention ist zudem um eine Einbindung der Schwellen- und Entwicklungsländer bemüht. Es geht dabei um einen ,offenen, nicht bindenden Austausch von Ansichten, Informationen und Ideen“. Bis zur 13. Vertragsstaatenkonferenz Ende 2007 sollen die Themen „Nachhaltige Entwicklungsziele“, „Anpassung an den Klimawandel“ und „Potenzial von Technologien und Marktmechanismen" behandelt werden.

Auf der ersten Dialogsitzung in Bonn wurde in Statements auf alle genannten Themen eingegangen; man war sich aber bald der Notwendigkeit bewusst, die Themen in den folgenden Workshops stärker zu fokussieren. Wie dies gelingt und ob der "open dialogue“ dazu beitragen kann, eine Strategie hin zu langfristig wirksamer internationaler Zusammenarbeit angesichts des immer offensichtlicher werdenden Klimawandels zu entwickeln, werden die nachfolgenden Dialogsitzungen zeigen.

\subsection{Die Sitzung der Ad-hoc-Arbeitsgruppe zum Kioto-Protokoll: „Further Commitments for Annex I Parties"}

Ein weiterer Bestandteil des Montrealer Aktionsplans war die Vereinbarung, auf der Basis von Artikel 3.9 des Kioto-Protokolls die bisher für die Industriestaaten (,Annex I“-Länder) vereinbarten Reduktionsziele $\mathrm{zu}$ überprüfen und vor allem Ziele für eine zweite Verpflichtungsperiode $a b 2013$ festzulegen. Die erste Verpflichtungsperiode reicht von 2008 bis 2012. Zum Vorsitzenden der dazu eingerichteten Ad hoc Arbeitsgruppe (AWG) wurde Michael Zammit Cutajar gewählt, ein „Urgestein“ der Verhandlungen zur Klimarahmenkonvention. Seit 1991 war er Exekutivsekretär des Zwischenstaatlichen Verhandlungsausschusses für eine Rahmenkonvention über Klimawandel (INC), in welchem die Verhandlungen bis zur ersten Vertragsstaatenkonferenz in Berlin geführt wurden. Danach war er bis 2002 Leiter des permanenten Sekretariats der Konvention in Bonn.

Auf der ersten Sitzung der Ad-hocArbeitsgruppe in Bonn beschäftigte man sich insbesondere damit, das Arbeitsprogramm für die nächsten Jahre festzulegen. Es wurde u. a. vereinbart, sich in den Verhandlungen auf Verpflichtungen für die Industrieländer zu beschränken und auf schnellstem Wege (,expeditiously") zu weiteren Verpflichtungen zu kommen, um keine Lücke zwischen beiden Verpflichtungsperioden entstehen $\mathrm{zu}$ lassen. Einig war man sich darüber hinaus, dass es nötig sei, Informationen über ,scientific, technical and socio-economic topics" zu beschaffen und auszuwerten, um sich über das nötige Ausmaß zukünftiger Verpflichtungen klar zu werden. Es wurde daher auch beschlossen, während der kommenden zweiten Konferenz der Vertragsparteien (COP/MOP 2) im November 2006 in Nairobi einen entsprechenden Workshop unter Beteiligung des Intergovernmental Panel on Climate Change zu organisieren, auf dem man sich mit Themen wie der zu erwartenden Emissionsentwicklung, dem Potential von Politik und Technik zur Verminderung von Emissionen (,Mitigation“) und den Kosten und Vorteilen von Emissionsreduktionen beschäftigen will.

Im Zusammenhang mit der nötigen Schärfe neuer Verpflichtungen der Industrieländer wird sicherlich immer wieder das insbesondere von Deutschland und der EU vorgetragene Ziel diskutiert werden, den globalen Temperaturanstieg auf maximal zwei Grad Celsius gegenüber einem vorindustriellen Niveau zu begrenzen. Die Menge dessen, was tatsächlich eingespart werden kann, ist allerdings sehr begrenzt, 
da der wichtigste Emittent von Treibhausgasen, die USA, das Kioto-Protokoll nicht ratifiziert hat und Länder mit einem starken Anstieg der Treibhausgasemissionen (wie China, Indien und Südkorea) nicht in die von der AWG diskutierten Verpflichtungen einbezogen werden sollen. Die $\mathrm{CO}_{2}$-Emissionen der Länder, über die man verhandelt, betragen derzeit weniger als 28 Prozent der globalen Emissionen; 1990 lag der Anteil noch bei 40 Prozent (mit den USA und anderen nicht am Kioto-Protokoll beteiligten Industriestaaten bei 64 Prozent). ${ }^{1}$

\subsection{Die Sitzung der Nebenorgane: "Subsidiary bodies"}

Die beiden Nebenorgane oder Subsidiary bodies SBI (Subsidiary Body for the Implementation of the United Nations Framework Convention on Climate Change) und SBSTA (Subsidiary Body for Scientific and Technological Advice) sind kein Produkt des Kioto-Protokolls, sondern Bestandteil der UN-Klimarahmenkonvention seit 1995. Der SBI hat dafür zu sorgen, dass die Weisungen der Klimarahmenkonvention und Beschlüsse der Vertragsstaatenkonferenzen effizient ausgeführt werden können. Die Funktion des SBSTA besteht im Wesentlichen darin, eine Verbindung zwischen den vorhandenen wissenschaftlichen Erkenntnissen und den vorwiegend politisch orientierten Bedürfnissen der Vertragsstaatenkonferenzen herzustellen. In Bonn kamen beide Nebenorgane zum 24. Mal zusammen.

$\mathrm{Zu}$ den Themen der Sitzung des SBI gehörten die Berichte („Communications“) der „Annex I"-Staaten, die bis 2005 abzugeben waren und Fortschritte auf dem Weg hin zu den KiotoZielen aufzeigen sollten. Die Abgabe der Berichte verlief bislang sehr schleppend, und von den „Nicht-Annex I“-Ländern (G77 / China) wurde gemutmaßt, dass die Industrieländer ihre Verpflichtungen nicht erfüllen würden (wozu außer den Emissionsreduktionen selbst auch die vorgeschriebene Berichterstattung gehört, d. A.). Drei weitere Themen wurden behandelt: die Berichterstattung der „Nicht-Annex I“-Länder, die finanziellen Mechanismen der Konvention und des Kioto-Protokolls (bei letzterem ging es insbesondere um den sog. Adaptation Fund, wo die Verhandlungen von Karsten Sach aus Deutschland als Co-Chair geleitet wurden), sowie die Implementation der Artikel 4.8 und 4.9 der Klimarahmenkonvention, in denen es um Hilfe für die besonders vom Klimawandel betroffenen Entwicklungsländer und finanzielle Unterstützung der ärmsten Länder geht.

Auf der Sitzung des SBSTA beschäftigte man sich zunächst mit einem Arbeitsprogramm für die nächsten fünf Jahre zum Thema „Anpassung an Klimaänderungen“, ohne sich jedoch darüber einig zu werden. Es ging des Weiteren um das Thema „Technologietransfer“, wobei wiederum auf die Wichtigkeit von Technologien hingewiesen wurde, die bei der Anpassung an Klimaänderungen eingesetzt werden können. Ein weiterer Punkt war die „Verminderung von Emissionen“, die durch Entwaldungsmaßnahmen in den Entwicklungsländern verursacht werden. Hierzu wird es Ende August einen Workshop in Rom geben, dessen Ergebnisse auf der nächsten Sitzung des SBSTA diskutiert werden sollen. Ein umfangreicher Themenkomplex mit diversen Unterpunkten waren die so genannten „Methodological Issues“, die insbesondere die technischen Aspekte der Emissionsberichterstattung umfassten.

\section{Ergebnisse}

Zunächst fällt auf, dass auf den Sitzungen des SBI und des SBSTA in Bonn kaum Entscheidungen getroffen wurden und die Fortsetzung der Arbeit in den unterschiedlichen Aufgabengebieten auf das nächste Treffen, das parallel zu COP / MOP 2 in Nairobi stattfinden soll, verschoben wurde. Von den neu begonnen Gesprächen in den Dialogsitzungen und von der neu eingesetzten Ad-hoc-Arbeitsgruppe zur Fortentwicklung des Kioto-Protokolls waren wichtige Ergebnisse sicher nicht zu erwarten gewesen. Hier bleibt deren Einrichtung als Ergebnis der Veranstaltung in Bonn zu konstatieren und in Zukunft die Ergebnisse der Arbeit der neuen Organe genauer unter die Lupe zu nehmen.

Von Beobachtern der Verhandlungen wird erstaunt festgestellt, dass die in der Woche zuvor in Köln ausgerichtete "Carbon Expo" mehr Besucher hatte als die Bonner Veranstaltung. Groß ist vor allem das Interesse der Wirtschaft an einer Option wie dem „Emissionshandel", womit man innerhalb der EU derzeit die ersten praktischen Erfahrungen sammelt und den man gerne weiter ausgedehnt sähe, 
aber auch an der technischen Option der Kohlenstoffabscheidung und -speicherung, die es möglich machen würde, mittelfristig auch weiterhin fossile Brennstoffe einzusetzen.

Es sieht so aus, als ob derzeit die Weichen für die Zukunft des Kioto-Protokolls und auch der Konvention selbst gestellt würden. Wird man sich in der Ad-hoc-Arbeitsgruppe über ambitionierte Reduktionsziele einig werden, wenn draußen in der Welt die größten Emittenten unbeschränkt immer mehr Treibhausgase emittieren? Ambitionierte Reduktionsziele für alle Industriestaaten wären ein wirksames Signal gewesen. Vielleicht können die Dialoggespräche den Horizont für ein weit gefasstes, alle Akteure umfassendes Klimaregime öffnen, wobei sich dieses derzeit schon durch das stark zunehmende Interesse der Wirtschaft an wirksameren Klimaschutzmaßnahmen anzudeuten scheint.

\section{Anmerkung}

1) Angaben laut DIW-Wochenbericht 39/2005

\section{Quellen}

UNFCCC-Sekretariat Bonn: Webcasts und Videos unter http://unfccc.int/meetings/webcast_and_videos/ items/2662.php

IISD - International Institute for Sustainable Development: Earth Negotiations Bulletin - ENB - unter: http://www.iisd.ca/climate/sb24/

CAN - The Climate Action Network: Climate Conference Newsletter - ECO - unter: http://www. climatenetwork.org/eco/

Weitere Informationsquellen bietet die vom Autor betreute Linksammlung, die ITAS unter dem Titel "Global Change and Climate Change Links" auf seiner Homepage im Internet anbietet: http://www.itas.fzk.de/deu/InfUm/infumd.htm

\section{Arbeit und Geschlecht im Um- bruch der modernen Gesell- schaft. Forschung im Dialog}

\author{
Dortmund, 28. - 29. April 2006
}

\section{Bericht von der gemeinsamen Tagung der Sektionen „Frauen- und Geschlechter- forschung" und „Arbeits- und Industrie- soziologie" der Deutschen Gesellschaft für Soziologie}

\section{von Linda Nierling und Bettina-Johanna Krings, ITAS}

\section{Hintergrund}

Welche Auswirkungen haben die aktuellen Entwicklungen auf dem Arbeitsmarkt für die Zukunft der Erwerbsarbeit? Wie wirken sich die gegenwärtigen wirtschaftlichen Rahmenbedingungen auf die Organisation von Arbeit in Unternehmen aus? Sind die Konsequenzen für Frauen und Männer unterschiedlich? Mit diesen Fragen beschäftigte sich die Tagung „Arbeit und Geschlecht im Umbruch der modernen Gesellschaft. Forschung im Dialog“ der "Sektionen Frauen- und Geschlechterforschung“ und „Arbeits- und Industriesoziologie" der Deutschen Gesellschaft für Soziologie. Sie fand vom 28. bis 29. April in Dortmund statt und wurde von der Sozialforschungsstelle Dortmund ausgerichtet. Mehr als 100 Wissenschaftlerinnen und Wissenschaftler diskutierten ausgesprochen leidenschaftlich über aktuelle Entwicklungen in der Erwerbsarbeit und deren verändernde Effekte auf das Geschlechterverhältnis.

Die Qualität der Diskussionen war ohne Zweifel durch die Besonderheit dieser Tagung geprägt: Es war historisch die erste gemeinsame Tagung der beiden Sektionen; sie hatte den Anspruch, den Dialog zwischen den beiden Diskussionssträngen $\mathrm{zu}$ fördern. In der Vergangenheit wurden wenige thematische Gemeinsamkeiten zwischen den beiden Richtungen gemeinsam diskutiert, was sicherlich an Anerkennungsproblemen der Frauen- und Geschlechterforschung innerhalb der Zunft selbst gelegen hat. Der Wunsch nach Dialog entstand offensichtlich in einer inhaltlichen Annäherung, da sich in den vergangenen Jahren die Arbeits- und Industriesoziologie zunehmend 